Communications in Physics, Vol.20, No. 1 (2010), pp. 15-21

\title{
SQUARKS DECAY INTO QUARKS AND GLUINOS IN THE MSSM WITH COMPLEX PARAMETERS
}

\author{
HA HUY BANG, NGUYEN THI THU HUONG \\ Hanoi University of Science, Vietnam National University Hanoi \\ NGUYEN CHINH CUONG \\ Hanoi University of Education
}

\begin{abstract}
We discuss the decay of squarks (stop and sbottom) into quarks (top and bottom) and gluinos in the Minimal Supersymmetric Standard Model (MSSM) with complex parameters $A_{t}$, $A_{b}$. The analytical and numerical results for the decay widths are given. We show that the effect of these CP phases on the decay widths of stop and sbottom decay can be quite significant in a large region of the MSSM parameter space. SUSY-QCD corrections to the decay widths have been included.
\end{abstract}

\section{INTRODUCTION}

The Minimal Supersymmetric Standard Model (MSSM) is considered the most attractive extension of the Standard Model. Many phenomenological studies on SUSY particle searches have been performed in the MSSM with real SUSY parameters. In general, however, some of the SUSY parameters may be complex, in particular the higgsino mass parameter $\mu$, the gaugino mass parameters $M_{1,2,3}$ and the trilinear scalar coupling parameters $A_{f}$ of the sfermions $\tilde{f}$, thus inducing explicit CP violation in the model. The $S U(2)$ gaugino mass parameter $M_{2}$ can be chosen real after an appropriate redefinition of the fields. Not only the CP-violating observables (such as fermion EDMs) but also the CPconserving observables (such as cross sections and decay branching ratios) depend on the phases of the complex parameters, because in general the mass-eigenvalues and the couplings of the SUSY particles involved are functions of the underlying complex parameters. For example, the decay branching ratios of the staus $\tilde{\tau}_{1,2}$ and $\tau$-sneutrino $\tilde{\nu}_{\tau}$ can be quite sensitive to the complex phases of the stau and gaugino-higgsino sectors [1]. Therefore, in a complete phenomenological analysis of production and decays of the SUSY particles one has to take into account that $A_{f}, \mu$ and $M_{1,3}$ can be complex.

In the MSSM every quark has two scalar partners, the squarks $\tilde{q}_{L}$ and $\tilde{q}_{R}$, corresponding to the left and the right helicity states of a quark. In general $\tilde{q}_{L}$ and $\tilde{q}_{R}$ mix to form mass eigenstates $\tilde{q}_{1}$ and $\tilde{q}_{2}$ (with $m_{\tilde{q}_{1}}<m_{\tilde{q}_{2}}$ ), the size of the mixing being proportional to the mass of the corresponding quark $q[2]$, and so neligible except for the third generation. Studies of the third generation squarks are particularly interesting because of the effects of the large Yukawa couplings. A number of studies of squarks production and decay within the MSSM with complex parameters are also available: the effect of the $\mathrm{CP}$ phases on the cross sections of squarks prodution at $e^{+} e^{-}$and $\mu^{+} \mu^{-}$colliders has 
been presented in $[3,4]$ with SUSY-QCD corrections included. CP phase dependences of the stop and sbottom $\left(\tilde{t}_{1,2}\right.$ and $\left.\tilde{b}_{1,2}\right)$ partial decay widths and branching ratios have been investigated for the fermionic decays

$$
\begin{gathered}
\tilde{q}_{i} \rightarrow q^{\prime}+\tilde{\chi}_{k}^{ \pm}, i=1,2, k=1,2 \\
\tilde{q}_{i} \rightarrow q+\tilde{\chi}_{k}^{0}, i=1,2, k=1, . ., 4
\end{gathered}
$$

in $[5,6,7,8]$ and for the bosonic decays

$$
\begin{gathered}
\tilde{q}_{i} \rightarrow \tilde{W}^{ \pm}+\tilde{q}_{j}^{\prime}, i=1,2, j=1,2, j \leq i \\
\tilde{q}_{i} \rightarrow \tilde{H}^{ \pm}+\tilde{q}_{j}^{\prime}, i=1,2, j=1,2, j \leq i \\
\tilde{q}_{2} \rightarrow Z+\tilde{q}_{1},
\end{gathered}
$$

and

$$
\tilde{q}_{2} \rightarrow H_{i}+\tilde{q}_{1}, i=1,2,3 .
$$

in $[5,6,7,9,10]$. Stop and sbottoms can also decay strongly through $\tilde{t}_{i} \rightarrow t \tilde{g}$ and $\tilde{b}_{i} \rightarrow b \tilde{g}$. If stops and sbottoms are very heavy and the gluinos mass is not too large, these decays are kinematically possible and important. Note that the decay $\tilde{t}_{1}\left(\tilde{b}_{1}\right) \rightarrow t(b) \tilde{g}$ may not be kinematically allowed. In this paper we study the effects of the phases of the complex parameters $A_{t, b}$ on the decay widths of the processes: $\tilde{t}_{2} \rightarrow t \tilde{g}$ and $\tilde{b}_{2} \rightarrow b \tilde{g}$. We find that these effects can be quite strong in a large region of the MSSM parameter space. This could have an important impact on the search for squarks and the determination of the MSSM parameters at future colliders. Our present study is an extension of the corresponding one in the MSSM with real parameters in [11, 12].

\section{TREE-LEVEL FORMULAE AND NOTATIONS}

In the MSSM the squark sector is specified by the mass matrix in the basis $\left(\tilde{q}_{L}, \tilde{q}_{R}\right)$ with $\tilde{q}=\tilde{t}$ or $\tilde{b}$

$$
\mathcal{M}_{\widetilde{q}}^{2}=\left(\begin{array}{cc}
m_{\widetilde{q}_{L}}^{2} & a_{q}^{*} m_{q} \\
a_{q} m_{q} & m_{\widetilde{q}_{R}}^{2}
\end{array}\right)
$$

with

$$
\begin{aligned}
m_{\widetilde{q}_{L}}^{2} & =M_{\widetilde{Q}}^{2}+m_{Z}^{2} \cos 2 \beta\left(I_{3}^{q_{L}}-e_{q} \sin ^{2} \theta_{W}\right)+m_{q}^{2}, \\
m_{\widetilde{q}_{R}}^{2} & \left.=M_{\{\widetilde{U}, \widetilde{D}\}}^{2}+m_{Z}^{2} \cos 2 \beta e_{q} \sin ^{2} \theta_{W}\right)+m_{q}^{2}, \\
a_{q} & =A_{q}-\mu\{\cot \beta, \tan \beta\} .
\end{aligned}
$$

Here $I_{3}^{q}$ is the third component of the weak isospin and $e_{q}$ the electric charge of the quark q. $M_{\tilde{Q}, \tilde{U}, \tilde{D}}$ and $A_{t, b}$ are soft SUSY-breaking parameters and $\tan \beta=v_{2} / v_{1}$ with $v_{1}\left(v_{2}\right)$ being the vacuum expectation value of the Higgs field $H_{1}^{0}\left(H_{2}^{0}\right)$. We take $A_{q}(q=t, b)$ as complex parameter: $A_{q}=\left|A_{q}\right| e^{i \phi_{A_{q}}}$ with $-\pi \leq \phi_{A_{q}} \leq \pi$. The current eigenstates $\tilde{q}_{L}$ and $\tilde{q}_{R}$ are related to their mass eigenstates $\tilde{q}_{1}$ and $\tilde{q}_{2}$ by

$$
\left(\begin{array}{c}
\widetilde{q}_{1} \\
\widetilde{q}_{2}
\end{array}\right)=R^{\widetilde{q}}\left(\begin{array}{c}
\widetilde{q}_{L} \\
\widetilde{q}_{R}
\end{array}\right),
$$


with complex parameters, we have

$$
R^{\widetilde{q}}=\left(\begin{array}{cc}
e^{i \phi_{\widetilde{q}}} \cos \theta_{\widetilde{q}} & \sin \theta_{\widetilde{q}} \\
-\sin \theta_{\widetilde{q}} & e^{-i \phi_{\widetilde{q}} \cos \theta_{\widetilde{q}}}
\end{array}\right)
$$

In the $\left(\tilde{q}_{1}, \tilde{q}_{2}\right)$ basis the squark interations with quarks and gluinos are given by

$$
L_{q \tilde{q} \tilde{g}}=-\sqrt{2} g_{s} T_{r s}^{a}\left[\bar{q}_{r}\left(R_{i 1}^{\tilde{q}} P_{R}-R_{i 2}^{\tilde{q}} P_{L}\right) \tilde{g}^{a} \tilde{q}_{i, s}+\overline{\tilde{g}}^{a}\left(R_{i 1}^{\tilde{q}} P_{L}-R_{i 2}^{\tilde{q}} P_{R}\right) q_{r} \tilde{q}_{i, s}^{*}\right] .
$$

At tree level, the amplitude of a squark decay into a quark and a gluino has the general form

$$
M^{0}\left(\widetilde{q}_{i} \rightarrow q+\widetilde{g}\right)=-\bar{u}\left(k_{2}\right) \sqrt{2} i g_{s} T_{r s}^{a}\left(R_{i 1}^{\widetilde{q}} P_{R}-R_{i 2}^{\widetilde{q}} P_{L}\right) v\left(k_{3}\right) .
$$

with $k_{1}, k_{2}$ and $k_{3}$ are the four-momenta of $\widetilde{q}_{i}, q$ and $\widetilde{g}$, respectively (Fig1.a). The tree level decay width can thus be written as

$\Gamma^{0}\left(\widetilde{q}_{i} \rightarrow q+\widetilde{g}\right)=\frac{4 \varepsilon k\left(m_{q}^{2}, m_{\widetilde{q}_{i}}^{2}, m_{\widetilde{g}}^{2}\right)}{16 \pi m_{\tilde{q}_{i}}^{3}}\left\{\left(\left|R_{i 1}^{\widetilde{q}}\right|^{2}+\left|R_{i 2}^{\widetilde{q}}\right|^{2}\right)\left(m_{\widetilde{q}_{i}}^{2}-m_{q}^{2}-m_{\widetilde{g}}^{2}\right)+m_{q} m_{\widetilde{g}} 2 \Re\left(R_{i 1}^{+\widetilde{q}} R_{i 2}^{\widetilde{q}}\right)\right\}$,

with $k^{2}\left(m_{q}^{2}, m_{\widetilde{q}_{i}}^{2}, m_{\widetilde{g}}^{2}\right)=m_{q}^{4}+m_{\widetilde{q}_{i}}^{4}+m_{\widetilde{g}}^{4}-2 m_{q}^{2} m_{\widetilde{q}_{i}}^{2}-2 . m_{q}^{2} m_{\widetilde{g}}^{2}-2 m_{\widetilde{q}_{i}}^{2} m_{\widetilde{g}}^{2}$ and $\varepsilon=4 \pi \alpha_{s} / 3$, which in the case of real parameters reduces to [13]

$$
\left.\Gamma^{0}\left(\widetilde{q}_{1,2} \rightarrow q+\widetilde{g}\right)=\frac{2 \alpha_{s} k\left(m_{q}^{2}, m_{\widetilde{q}_{1,2}}^{2}, m_{\widetilde{g}}^{2}\right)}{3 m_{\tilde{q}_{1,2}}^{3}}\left(m_{\widetilde{q}_{1,2}}^{2}-m_{q}^{2}-m_{\widetilde{g}}^{2}\right) \pm 2 \sin 2 \theta_{\tilde{q}} m_{q} m_{\widetilde{g}}\right),
$$

where the "+" sign corresponds to $\tilde{q}_{1}$ decay and the "-" sign corresponds to $\tilde{q}_{2}$ decay.

\section{III. . SUSY-QCD CORRECTIONS}

The $\mathrm{O}\left(\alpha_{s}\right)$ loop correction to the decay width is

$$
\delta \Gamma=\delta \Gamma^{(v)}+\delta \Gamma^{(w)}+\delta \Gamma^{(c)}+\delta \Gamma_{\text {real }}=\frac{k\left(m_{q}^{2}, m_{\widetilde{q}_{i}}^{2}, m_{\tilde{g}}^{2}\right)}{16 \pi m_{q}^{3}} \cdot 2 \Re\left(M . \delta M^{+}\right),
$$

where the superscript $w$ denotes the wave function correction (Fig 1.b), $v$ denotes the vertex correction (Fig 1.c), c the counterterm to the couplings, and real the real gluon emission (Fig 1.c) which is included in order to cancel the infrared divergence. And

$$
\begin{aligned}
M . \delta M^{+}= & 4 \varepsilon\left\{\left[\left|R_{i 1}^{\widetilde{q}}\right|^{2}+\left|R_{i 2}^{\widetilde{q}}\right|^{2}\right]\left(m_{\widetilde{q}_{i}}^{2}-m_{q}^{2}-m_{\widetilde{g}}^{2}\right)+2 m_{q} m_{\widetilde{g}} \Re\left(R_{i 1}^{+\widetilde{q}} R_{i 2}^{\widetilde{q}}\right)\right\}\left(I_{1}+I_{2}+I_{3}\right)^{+} \\
& +2 \varepsilon\left(I_{4}+I_{5}\right)^{+}\left[R_{i 1}^{\widetilde{q}}-R_{i 2}^{\widetilde{q}}\right]\left(m_{\widetilde{q}_{i}}^{2}-m_{q}^{2}-m_{\widetilde{g}}^{2}-4 m_{q} m_{\widetilde{g}}\right),
\end{aligned}
$$


(a)

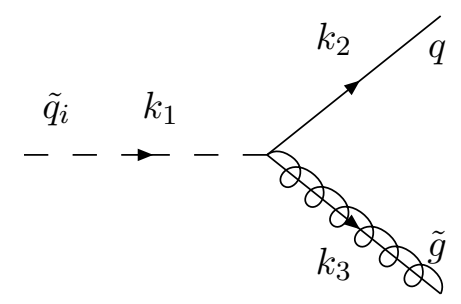

(b)
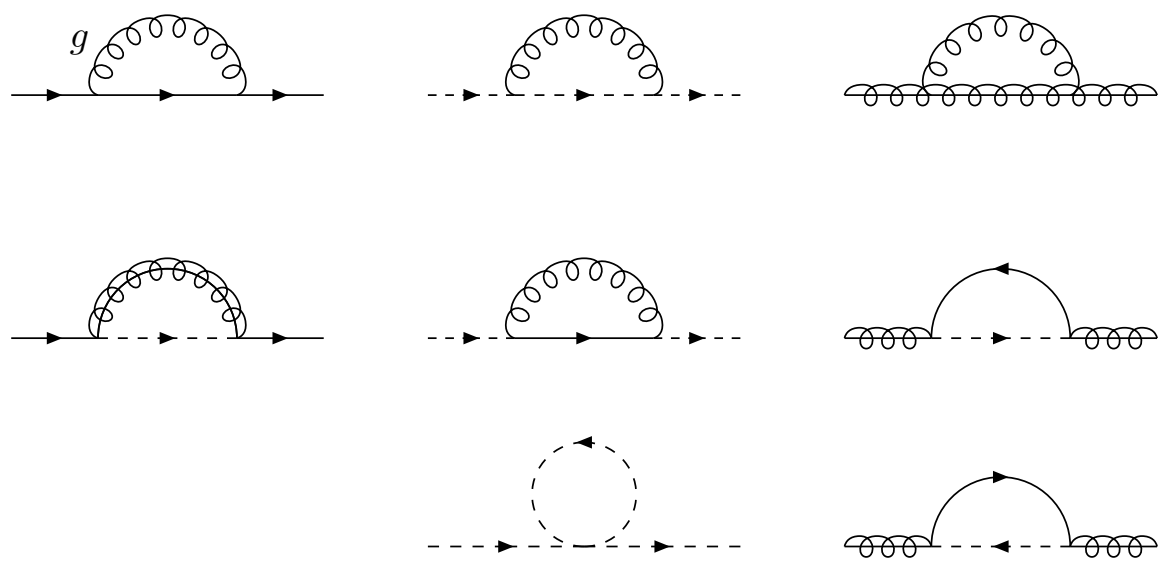

(c)
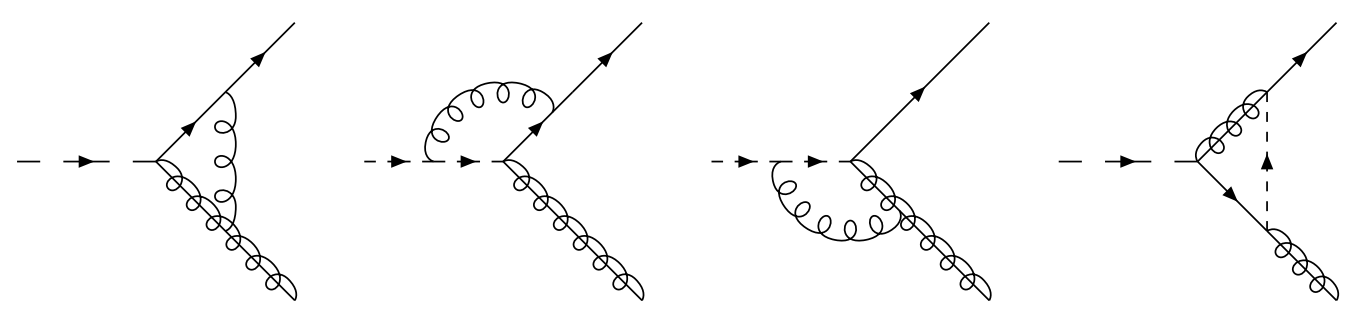

(d)
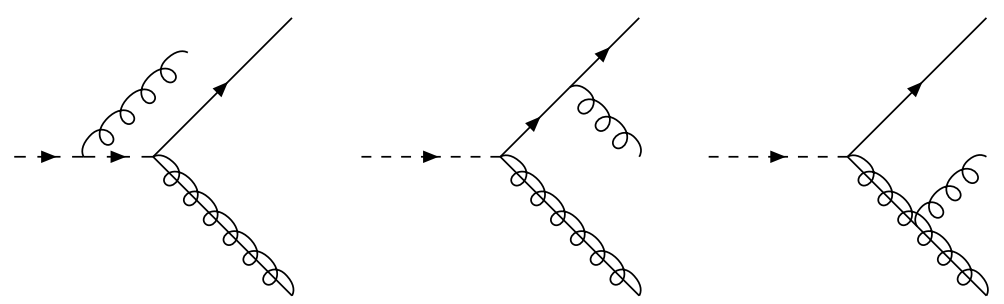

Fig. 1. Feynman diagrams for the $\mathrm{O}\left(\alpha_{s}\right)$ SUSY-QCD corrections to squarks decays into quarks and gluinos: (a) tree level; (b) quark, squark, and gluino selfenergies; and (c) vertex corrections, (d) real gluon emission. 
with

$$
\begin{aligned}
I_{1}= & \frac{-i \varepsilon}{16 \pi^{2}}\left[A_{0}\left(m_{\widetilde{q}_{i}}^{2}\right)+4 m_{\widetilde{q}_{i}}^{2} B_{0}\left(m_{\widetilde{q}_{i}}^{2}, 0, m_{\widetilde{q}_{i}}^{2}\right)+4 m_{\widetilde{q}_{i}}^{2} B_{1}\left(m_{\widetilde{q}_{i}}^{2}, 0, m_{\widetilde{q}_{i}}^{2}\right)\right] \\
I_{2}= & \frac{4 i \cdot \varepsilon}{16 \pi^{2}}\left\{A_{0}\left(m_{q}^{2}\right)+m_{\widetilde{q}_{i}}^{2} B_{1}\left(m_{\widetilde{q}_{i}}^{2}, m_{\widetilde{g}}^{2}, m_{q}^{2}\right)+\right. \\
& \left.+\left[m_{\widetilde{g}}^{2}+\left(R_{11}^{\widetilde{q}} \cdot R_{i 2}^{\widetilde{q}}+R_{21}^{\widetilde{q}} R_{i 2}^{\widetilde{q}}+R_{12}^{\widetilde{q}} R_{i 1}^{\widetilde{q}}+R_{22}^{\widetilde{q}} R_{i 1}^{\widetilde{q}}\right) m_{\widetilde{g}} m_{q}\right] \cdot B_{0}\left(m_{\widetilde{q}_{i}}^{2}, m_{\widetilde{g}}^{2}, m_{q}^{2}\right)\right\}, \\
I_{3}= & \frac{\varepsilon}{16 \pi^{2}}\left(S_{k n}^{\alpha} S_{i n}^{\alpha}+S_{k n}^{\alpha} S_{i n}^{\beta} \delta_{\alpha \beta}\right) A_{0}\left(m_{\widetilde{q}_{n}}^{2}\right), \\
I_{4}= & \frac{2 g_{s}^{2} T_{s s^{\prime}}^{a} f_{a b c}}{16 \pi^{2}}\left(R_{i 1}^{\widetilde{q}}-R_{i 2}^{\widetilde{q}}\right)\left\{B_{0}\left(m_{q}^{2}, m_{\widetilde{g}}^{2}, m_{\widetilde{q}_{i}}^{2}\right)+B_{0}\left(m_{\widetilde{q}_{i}}^{2}, m_{\widetilde{g}}^{2}, m_{\widetilde{q}_{i}}^{2}\right)+\right. \\
& +2\left(m_{\widetilde{g}}^{2}+m_{\widetilde{q}_{i}}^{2}-m_{q}^{2}\right)\left[C_{11}\left(m_{\widetilde{g}}^{2}, m_{\widetilde{q}_{i}}^{2}, m_{q}^{2}, 0, m_{\widetilde{g}}^{2}, m_{\widetilde{q}_{i}}^{2}\right)+C_{0}\left(m_{\widetilde{g}}^{2}, m_{\widetilde{q}_{i}}^{2}, m_{q}^{2}, 0, m_{\widetilde{g}}^{2}, m_{\widetilde{q}_{i}}^{2}\right)\right]+ \\
& \left.+2\left(m_{\widetilde{g}}^{2}-m_{\widetilde{q}_{i}}^{2}+m_{q}^{2}\right) C_{22}\left(m_{\widetilde{g}}^{2}, m_{\widetilde{q}_{i}}^{2}, m_{q}^{2}, 0, m_{\widetilde{g}}^{2}, m_{\widetilde{q}_{i}}^{2}\right)\right\}, \\
I_{5}= & \frac{g_{s}^{2} T_{s s^{\prime}}^{a} f_{a b c}}{16 . \pi^{2}} \cdot\left(R_{i 1}^{\widetilde{q}}-R_{i 2}^{\widetilde{q}}\right)\left[-B_{0}\left(m_{q}^{2}, 0, m_{q}^{2}\right)-B_{0}\left(m_{\widetilde{g}}^{2}, 0, m_{\widetilde{g}}^{2}\right)+\right. \\
& \left.+2\left(m_{\widetilde{g}} m_{q}+m_{q}^{2}\right) C_{0}\left(m_{\widetilde{g}}^{2}, m_{\widetilde{q}_{i}}^{2}, m_{q}^{2}, 0, m_{\widetilde{g}}^{2}, m_{q}^{2}\right)\right] .
\end{aligned}
$$

where

$$
\begin{aligned}
A_{0}\left(m^{2}\right) & =\int \frac{d^{4} q}{i \pi^{2}} \frac{1}{\left(q^{2}-m^{2}+i \varepsilon\right)}, \\
B_{0 ; \mu}\left(p^{2}, m_{1}^{2}, m_{2}^{2}\right) & =\int \frac{d^{4} q}{i \pi^{2}} \frac{1 ; q_{\mu}}{\left(q^{2}-m_{1}^{2}+i \varepsilon\right)\left[(q+p)^{2}-m_{2}^{2}+i \varepsilon\right]}, \\
B_{\mu}\left(p^{2}, m_{1}^{2}, m_{2}^{2}\right) & =p_{\mu} \cdot B_{1}\left(p^{2}, m_{1}^{2}, m_{2}^{2}\right) . \\
C_{0 ; \mu ; \mu \nu} & \equiv C_{0 ; \mu ; \mu \nu}\left(p^{2}, k^{2},(p+k)^{2}, m_{1}^{2}, m_{2}^{2}, m_{3}^{2}\right) \\
= & \int \frac{d^{4} q}{i \pi^{2}} \frac{1 ; q_{\mu} ; q_{\mu \nu}}{\left(q^{2}-m_{1}^{2}+i \varepsilon\right)\left[(q+p)^{2}-m_{2}^{2}+i \varepsilon\right]\left[(q+p+k)^{2}-m_{3}^{2}+i \varepsilon\right]}, \\
C_{\mu} & =p_{\mu} \cdot C_{11}+k_{\mu} \cdot C_{12}, \\
C_{\mu \nu} & =p_{\mu} p_{\nu} \cdot C_{21}+k_{\mu} k_{\nu} \cdot C_{22}+\{p k\}_{\mu \nu} \cdot C_{23}+\delta_{\mu \nu} \cdot C_{24},
\end{aligned}
$$

Thus the $\mathrm{O}\left(\alpha_{s}\right)$ corrected decay width $\Gamma$ is given by

$$
\Gamma=\Gamma^{0}+\delta \Gamma \text {. }
$$

where $\delta \Gamma$ is obtained from eqs. (10), (11) and (12).

\section{NUMERICAL RESULTS AND DISCUSSIONS}

Let us now turn to the numerical analysis. The squarks masses and mixing angles are fixed by the assumptions $M_{\widetilde{D}}=1.12 M_{\widetilde{Q}},\left|A_{t}\right|=\left|A_{b}\right|=|A|$ and $\cos \theta_{\tilde{t}}=-0.5$, $\cos \theta_{\tilde{b}}=-0.9$. We take $m_{t}=175 \mathrm{GeV}, m_{b}=5 \mathrm{GeV}$ and $\phi_{1}=\phi_{\mu}=0$. In our numerical study we take $\tan \beta, m_{\tilde{t}_{1}}, m_{\tilde{t}_{2}}, m_{\tilde{b}_{1}}, m_{\tilde{b}_{2}}, m_{\tilde{g}},|A|,|\mu|, \phi_{A_{t}}$ and $\phi_{A_{b}}$ and $\cos \theta_{\tilde{t}, \tilde{b}}$ as input parameters, where $m_{\tilde{t}_{1,2}}, m_{\tilde{b}_{1,2}}$ are the on-shell squark masses.

We make a comparision with the decay widths at tree-level and one-loop level for the real parameters case by studying the $\phi_{A_{t, b}}$ dependence of the ratios $\Gamma_{R}^{0} / \Gamma^{0}, \Gamma_{R} / \Gamma$, with $R$ 
indice corresponding to the case of real parameters. The input parameters are: $\tan \beta=3$, $m_{\tilde{t}_{1}}=350 \mathrm{GeV}, m_{\tilde{t}_{2}}=650 \mathrm{GeV}, m_{\tilde{b}_{1}}=170 \mathrm{GeV}, m_{\tilde{b}_{2}}=520 \mathrm{GeV}, m_{\tilde{g}}=500 \mathrm{GeV},|A|=$ $300 \mathrm{GeV},|\mu|=300 \mathrm{GeV}$. The experimental limits on the EDMs of electron, neutron, $\mathrm{Hg}$ and $T l$ strongly constrain the SUSY CP phases [14]. In some scenarios $[15,16]$ the $\phi_{A_{t, b}}$, $\phi_{\mu}$ and $\phi_{1}$ are practically unconstrained. Here we concentrate on the small range of the complex phase $\phi_{A_{\tilde{t}, \tilde{b}}}$.

Figure (2) shows the $\phi_{2}=\phi_{A_{b}}$ dependence of the ratios $\Gamma_{R}^{0} / \Gamma^{0}, \Gamma_{R} / \Gamma$ for the $\tilde{b}_{2}$ decay into $b$ and $\tilde{g}$. In the range of $\phi_{2}$ shown, the $\phi_{2}$ can contribute $\approx-1.4 \% \rightarrow 0 \%$ to the $\Gamma^{0}$ and $\approx-4.6 \% \rightarrow 0 \%$ to the $\Gamma$.
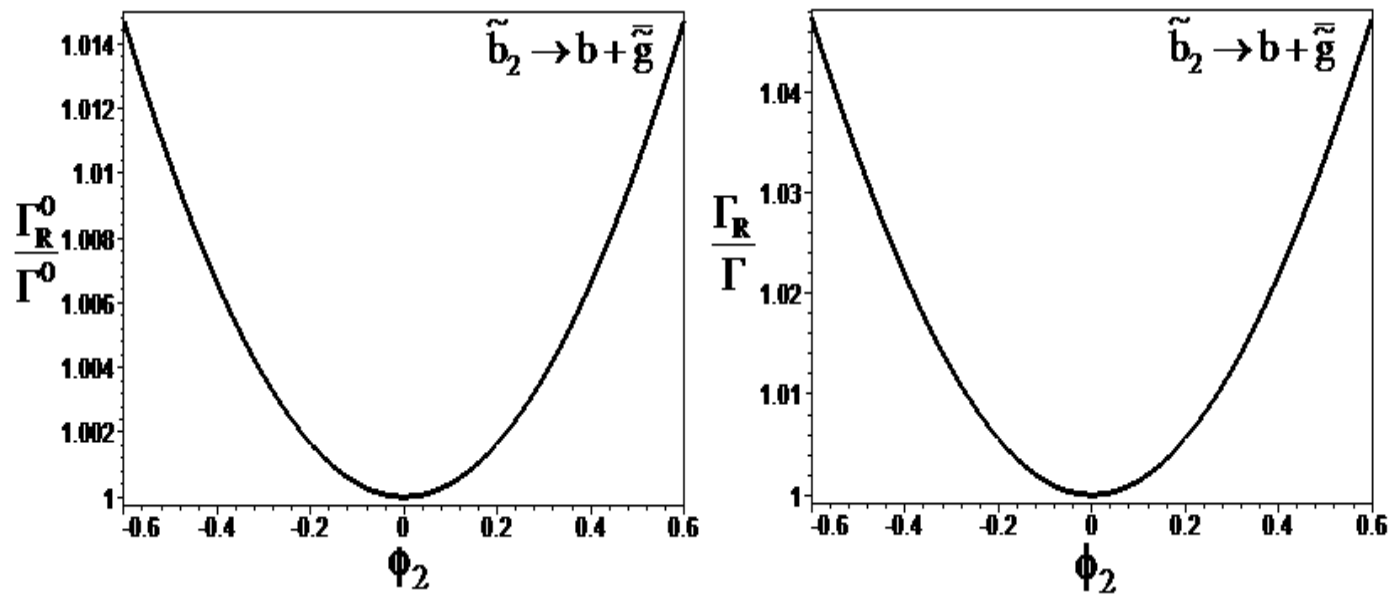

Fig. 2. The dependence of $\Gamma_{R}^{0} / \Gamma^{0}$ and $\Gamma_{R} / \Gamma$ on $\phi_{2}$ in the decays $\widetilde{b}_{2} \rightarrow b+\overline{\widetilde{g}}$ for $m_{\widetilde{g}}=500 \mathrm{GeV}, m_{\widetilde{b}_{2}}=520 \mathrm{GeV}, m_{\widetilde{b}_{1}}=170 \mathrm{GeV}$ and $\cos \theta_{\widetilde{b}}=-0.9$.
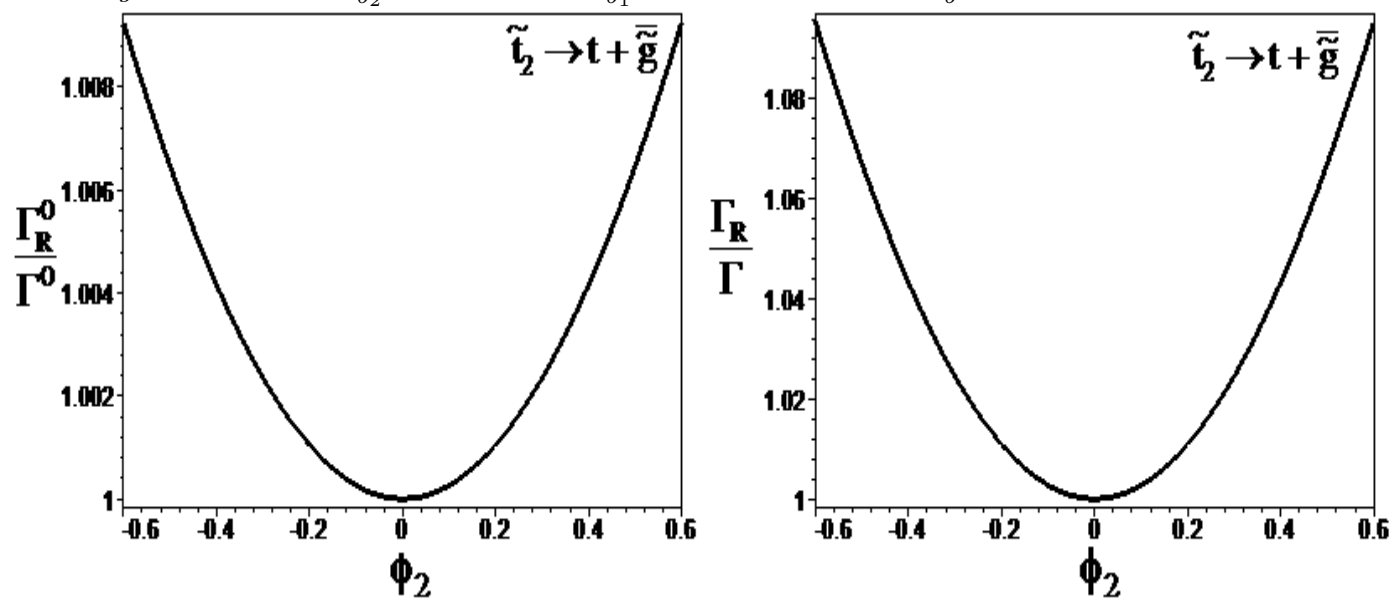

Fig. 3. The dependence of $\Gamma_{R}^{0} / \Gamma^{0}$ and $\Gamma_{R} / \Gamma$ on $\phi_{2}$ in the decays $\widetilde{t}_{2} \rightarrow t+\overline{\widetilde{g}}$ for $m_{\widetilde{g}}=500 \mathrm{GeV}, m_{\widetilde{t}_{2}}=650 \mathrm{GeV}, m_{\widetilde{t}_{1}}=350 \mathrm{GeV}$ and $\cos \theta_{\widetilde{t}}=-0.5$. 
Similarly, in figure (3) we show the $\phi_{2}=\phi_{A_{t}}$ dependence of the ratios $\Gamma_{R}^{0} / \Gamma^{0}, \Gamma_{R} / \Gamma$ for the $\tilde{t}_{2}$ decay into $t$ and $\tilde{g}$. As can be seen, the effect of $\phi_{2}=\phi_{A_{t}}$ on this process is stronger. In the range of $\phi_{2}$ shown, the $\phi_{2}$ can contribute $\approx-0.8 \% \rightarrow 0 \%$ to the $\Gamma^{0}$ and $\approx-9.5 \% \rightarrow 0 \%$ to the $\Gamma$.

\section{CONCLUSIONS}

We have studied the decays of squarks $\left(\tilde{t}_{2}, \tilde{b}_{2}\right)$ into quarks $(t, b)$ and gluinos in the MSSM with complex parameters $A_{t, b}$. We have shown that including CP phases strongly affect the decay widths of $\tilde{t}_{2}$ and $\tilde{b}_{2}$ decays in a large domain of the MSSM parameter space. This could have important implications on the search for $\tilde{t}_{i}$ and $\tilde{b}_{i}$ and the determination of the underlying MSSM parameters at future colliders.

\section{REFERENCES}

[1] A. Bartl, K. Hidaka, T. Kernreiter and W. Prod, Phys. Lett. B538 (2002) 137 [hep-ph/0204071]; Phys. Rev. D66 (2002) 115009 [hep-ph/0207186].

[2] J. Ellis, S. Rudaz, Phys. Lett. B128 (1983) 248; J. F. Gunion, H. E. Haber, Nucl. Phys. B272 (1986) 1; B402 (1993) 567 (E).

[3] N. T. T. Huong, H. H. Bang, N. C. Cuong and D. T. L. Thuy, Int. J. of Theor. Phys. 46(1) (2007) 41.

[4] N. T. T. Huong, N. C. Cuong, H. H. Bang and D. T. L. Thuy, Int. J. of Theor. Phys., DOI 10.1007/s10773-010-0326-1.

[5] A. Bartl, S. Hesselbach, K. Hidaka, T. Kernreiter and W. Prod, Phys. Lett. B573 (2003) 153 [hepph/0307317].

[6] A. Bartl, S. Hesselbach, K. Hidaka, T. Kernreiter and W. Prod, Phys. Rev. D70 (2004) 035003 [hep-ph/0311338].

[7] A. Bartl, S. Hesselbach, K. Hidaka, T. Kernreiter and W. Prod, [hep-ph/0409347].

[8] T. Ibrahim and P. Nath, [hep-ph/0411272].

[9] D. T. L. Thuy, N. C. Cuong and H. H. Bang, Comm. in Phys. 14 (2004) 76.

[10] N. C. Cuong and H. H. Bang, Comm. in Phys. 14 (2004) 23.

[11] W. Beenakker, R. Hopker and P. M. Zerwas, Phys. Lett. B378 (1996) 159 [hep-ph/9602378].

[12] W. Beenakker, R. Hopker, T. Plehn and P. M. Zerwas, Z. Phys. C75 (1997) 349 [hep-ph/9610313].

[13] W. Porod, PhD thesis [hep-ph/9804208].

[14] Review of Particle Physics, Eur. Phys. J. C3 (1998) 1-794.

[15] A. G. Cohen, D. B, Kaplan, A. E. Nelson, Phys. Lett., B388 (1996) 588; A. G. Akeroyd, Y. Y. Keum, S. Recksiegel, Phys. Lett., B507 (2001) 78.

[16] T. Ibrahim and P. Nath, Phys. Lett. B418 (1998) 98 [hep-ph/9707409]; Phys. Rev. D57 (1998) 478 [hep-ph/9708456]; Phys.Rev. D58 (1998) 111301 [hep-ph/9807501].

Received 01 Janunary 2009. 